\title{
Der Erweiterungsbau der WLB - Teil 2
}

Am 27. Mai 2011 hat sich das Preisgericht für den Entwurf des Stuttgarter Architekturbüros Lederer Ragnarsdóttir Oei (LRO) entschieden. Der Siegerentwurf sieht einen eigenständigen Erweiterungsbau vor, der in der ersten Bauphase ohne Störung des Bibliotheksbetriebs im Bestandsgebäude errichtet werden kann. In einem zweiten Bauabschnitt wird nach der Verlagerung von Leihstelle, Fernleihe, Selbstabholerbereich, Freihandmagazin, Information, Ausstellungsbereich, Vortragsraum und Cafeteria in den Erweiterungsbau das Bestandsgebäude entsprechend umgebaut, um die freigewordenen Flächen einer neuen Nutzung zuzuführen.

Zur Vorbereitung der Bauunterlage haben seit der Entscheidung des Preisgerichts 15 Nutzergespräche (Amt für Vermögen und Bau, LRO, Nutzer) und 12 Planungsbesprechungen (Amt für Vermögen und Bau, Fachplaner, LRO, Nutzer) stattgefunden. Am 20. August 2012 ist die Bauunterlage beim Ministerium für Finanzen und Wirtschaft (MFW) eingereicht worden.

Nach dem jetzt erreichten Planungsstadium sind die Funktionsbereiche der WLB wie folgt zwischen Erweiterungsbau und Bestandsgebäude aufgeteilt. In dem sechsgeschossigen Erweiterungsbau werden sämtliche

Funktionsbereiche mit hoher Benutzungsfrequenz untergebracht sein. Im Bestandsgebäude befinden sich die Lesebereiche für ruhiges und konzentriertes Arbeiten mit einem Präsenzbestand von ca. 250.000 Bänden, der Sonderlesesaal sowie die internen Bibliotheksabteilungen. Bestandsgebäude und Erweiterungsbau sind durch einen Steg im ersten Obergeschoss verbunden.
Die neue WLB verfügt über einen zentralen Haupteingang im Erweiterungsbau. In dem großzügig gestalteten Eingangsbereich sind sämtliche Funktionsbereiche für die schnelle Information sowie den Ausleihvorgang (Leihstelle, Selbstabholerbereich, Selbstverbuchung, Rückgabe- und Kassenautomat) untergebracht.

Von der Eingangshalle aus gelangt der Benutzer über eine elektronisch gesicherte Zugangskontrolle in den gesicherten Bereich der Bibliothek. Ziel ist eine Infrastruktur zu schaffen, die eine 24-Stunden-Bibliothek ermöglicht. Zunächst wird jedoch von Öffnungszeiten von 8.00 bis $24.00 \mathrm{Uhr}$ an sieben Tagen der Woche ausgegangen. Eine 24-Stunden-Öffnung wird vor allem unter wirtschaftlichen Aspekten zu prüfen sein.

Ebenfalls im EG untergebracht sind der Ausstellungsbereich und der Vortragssaal.

Auf der Höhe der Konrad-Adenauer-Straße mit direktem Zugang von dem geplanten "Boulevard" befinden sich die Cafeteria und Bar.

Der große Freihandbereich (ca. 500.000 Bände) mit attraktiven Lese- und Arbeitsplätzen an den Fensterseiten verteilt sich auf das 1. bis 4. Obergeschoss.
Mit

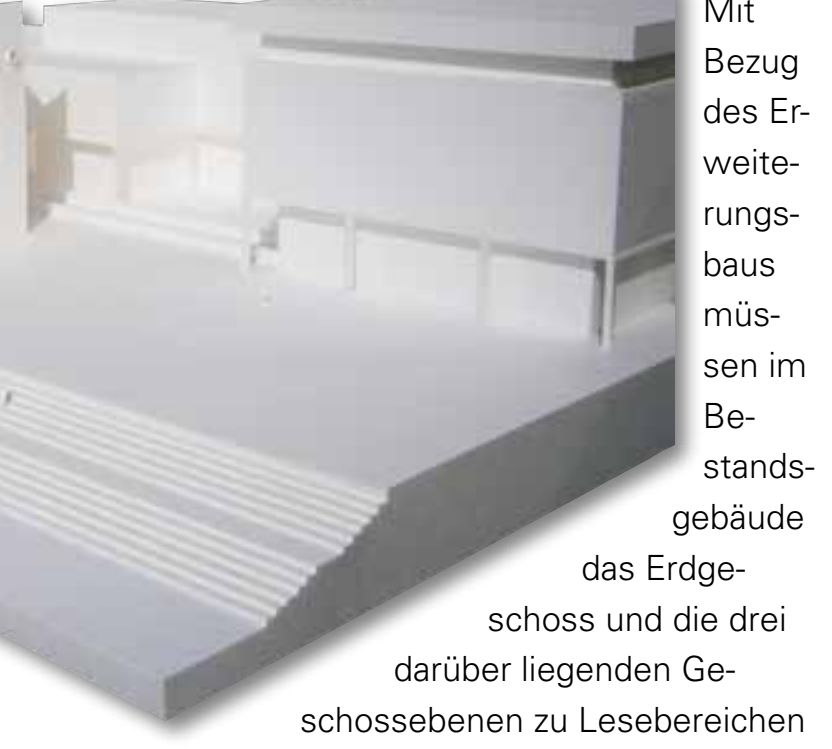

mit Präsenzbestand und Arbeitsplätzen

sowie Gruppenarbeitsplätzen umgebaut werden. 
Der neue Sonderlesesaal soll im Bereich des jetzigen Sonderlesesaales Alte Drucke, Handschriften und Kunst nach einem Komplettumbau entstehen. Der Hauptlesesaal, der nach 42 Jahren nichts von seiner Attraktivität verloren hat, bleibt für ruhiges und konzentriertes Arbeiten unverändert. Der Appendix mit Musiklesesaal und Vortragsraum soll abge-

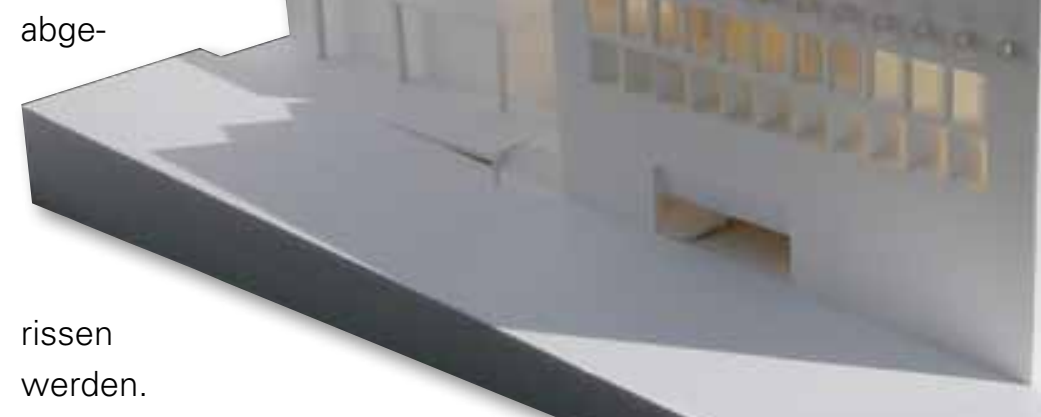

werden.
Magazine erfolgt konventionell mit Personal und Bücherwagen.

Das MFW hat nach eingehender

Prüfung aus wirtschaftlichen Erwägungen beschlossen, die bestehende Tiefgarage vor der Landesbibliothek abzubrechen und neu zu bauen. Dadurch können die notwendigen Flächen für die Technik und ein Tiefmagazin mit direkter Anbindung an die Tiefmagazine im Bestandsgebäude entsprechend dem Raumprogramm untergebracht werden.

Die neue Tiefgarage erhält die notwendige Infrastruktur für Tiefmagazine, sodass sie zu einem späteren Zeitpunkt auch als Büchermagazin genutzt werden kann. Dies ist bei der jetzigen Tiefgarage nicht möglich.

Für den Erweiterungsbau und das Bestandsgebäude ist ein RFID-gestütztes Buchfördersystem geplant. Die über 40 Jahre alte Buchförderanlage ist wegen ihrer Störanfälligkeit - Ersatzteile gibt es schon lange nicht mehr - den heutigen Anforderungen nicht mehr gewachsen. Derzeit ist der Buchtransport nur aus den Tiefmagazinen in die Leihstelle möglich. Der Rücktransport in die
Über einen Außenschalter an der Ulrichstraße ist künftig auch eine 24-Stunden-Buchrückgabe möglich.

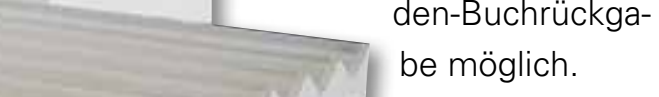
bleibt zu hoffen, dass nach Einreichen der Bauunterlage die Baukosten für den

Erweiterungsbau im Doppelhaushalt 2013/14 bewilligt werden.

Mit dem Abbruch der Tiefgarage soll im Sommer 2013 begonnen werden, sodass der Erweiterungsbau nach 20 Monaten Bauzeit 2015 zum 250-jährigen Jubiläum der Bibliothek in Betrieb genommen werden kann. Vor allem auch deshalb, da 2015 alle Raumreserven erschöpft sind und keine neuen Bücher mehr eingestellt werden können.

Ungeachtet der notwendigen Stellplatzerweiterung soll die neue WLB zu einem multimedialen Lernort werden mit herausragender Raumqualität und einer Vielfalt an Arbeitsmöglichkeiten. Dem „Wohlfühlfaktor" wird deshalb ein besonderer Stellenwert eingeräumt mit dem Ziel, einen Ort zu schaffen, der Lernen, Forschen, Lesen, Kommunikation und Arbeiten in der Bibliothek zu einem Erlebnis macht.

Hannsjörg Kowark 